\title{
Cost-Effectiveness Analysis of Complementary and Alternative Medicine in Treating Anxiety Disorders
}

Matthew Kutch*

Department of Economics, Ohio Northern University, USA

"Corresponding author: Matthew Kutch, Department of Economics, Ohio Northern University, USA, Tel: 419772-3929; E-mail: m-kutch@onu.edu

Received date: May 11, 2016; Accepted date: July 6, 2016; Published date: July 13, 2016

Copyright: ( 2016 Kutch M. This is an open-access article distributed under the terms of the Creative Commons Attribution License, which permits unrestricted use, distribution, and reproduction in any medium, provided the original author and source are credited.

\begin{abstract}
Objectives: Using survey data, this study produces estimates of the cost, effectiveness, and cost-effectiveness of complementary and alternative medicine combined with traditional therapies (pharmacotherapy and/or psychotherapy) in the treatment of anxiety disorders. Unlike past cost-effectiveness analyses that use a narrow definition or randomized controlled trials, this study uses a broad definition of complementary and alternative medicines and survey data to assess the cost-effectiveness.

Study Desi and Outcome Measures: This analysis uses the Medical Expenditure Panel Survey (MEPS), an overlapping panel survey of medical use, expenditure, and health status for the civilian non-institutionalized U.S. population to identity economic costs and effects for individuals with anxiety disorders. The primary measure of effect is based on self-perceived mental health status. Cost-effectiveness is determined by estimation of incremental net benefit method. Potential self-selection bias is investigated using observable characteristics and inverse propensity score weighting.

Results: Complementary and alternative medicine users with an anxiety disorder showed a statistically insignificant decrease in costs compared to nonusers ( $\$ 458.95$ versus $\$ 467.03 ; p$-value 0.920 ). Complementary and alternative medicine expenditures were offset by a statistically significant decrease in office-based and pharmaceutical costs. Complementary and alternative medicine users showed an increase in the probability of steady or improved mental health compared to nonusers (0.7549 versus 0.6912 ; $p$-value 0.190$)$ resulting in an incremental cost-effectiveness ratio of $-\$ 12.70$ per $10 \%$ increase in the probability of steady or increased mental health. The evidence suggests a high probability that complementary and alternative medicine is cost-effective for large values of effect for anxiety disorders ( $p$-value 0.080 for WTP of $\$ 5000$ ).
\end{abstract}

Conclusions: This survey data indicates complementary and alternative medicine users with anxiety disorders had slightly lower costs and improved outcomes. Limitations are considered.

Keywords: Cost; Cost-effectiveness; Economic evaluation; Anxiety

\section{Abbreviations:}

CAM: Complementary and Alternative Medicine; MEPS: Medical Expenditure Panel Survey; ICER: Incremental Cost-Effectiveness Ratio; INB: Incremental Net Benefit; WTP: Willingness-to-Pay

\section{Introduction}

Anxiety and depression have a significant global prevalence $(22.0 \%)$ and are associated with some of the most significant impacts on quality of life [1]. A large-scale nationally representative survey estimated 12month prevalence for any mental health disorder at over $25 \%$ of the US adult population with anxiety disorders and mood disorders (depression disorder and bipolar disorder) with particularly high rates of prevalence ( $18.1 \%$ and $9.5 \%$ respectively) [2]. US Spending on mental health and substance abuse is projected to reach $\$ 281$ billion by 2020 , representing $6.5 \%$ of all health spending [3].

Conventional treatments for anxiety disorders include medication or cognitive-behavioral therapy (CBT). Medications cannot cure anxiety disorders, but can keep them under control while receiving psychotherapy [4]. In CBT patients change their thinking patterns that support their fears and change the way they react to anxiety-provoking situations. Similar to many Complementary and alternative medicine (CAM) modalities, CBT may often incorporate deep-breathing exercises to relieve anxiety and encourage relaxation.

Complementary and alternative medicines (CAM) are forms of treatment that are used in addition to (complementary) or instead of (alternative) standard treatment and can include a wide array of treatments, from acupuncture and massage therapy to herbal remedies and hypnosis. Complementary and alternative medicine (CAM) covers a heterogeneous spectrum of ancient to new-age approaches that purport to prevent or treat disease [5]. Practices are considered unconventional when they are either not widely taught in medical schools or when they are not widely practiced in hospitals or outpatient facilities [6].

Many individuals use CAM to improve health and well-being, including the relief of symptoms associated with chronic illness or the side-effects to conventional treatment. A previous study used the 2007 National Health Interview Survey (NHIS) to describe patterns of CAM users' characteristics and use, finding $38 \%$ of adults had used complementary and alternative medicine in the previous 12 month, 
most commonly nonvitamin, nonmineral, natural products, deep breathing exercises, meditation, chiropractic or osteopathic manipulation, massage therapy, and yoga [5]. Common diseases and conditions for which people use CAM includes back, neck, or joint pain, arthritis, and mental health disorders (anxiety, depression, ADHA/ADD) [5].

Past studies demonstrate the increased use of CAM for individuals with mental health disorders. Kessler et al. [6] using a 1997-1998 nationally representative survey suggests that the majority of people in the United States with self-defined anxiety attacks or severe depression use some form of CAM as a component of treatment. This assumes a broadly drawn definition of CAM that includes prayer as spiritual healing, relaxation techniques, and imagery. The authors find no statistical difference in the observable characteristics of individuals with anxiety attacks or severe depression who use CAM and individuals without anxiety attacks or severe depression who use of CAM.

Unützer et al. [7] similarly found individuals who reported use of alternative medicine were more likely to meet diagnostic criteria for at least one of the mental disorders under consideration (depression, dysthymia, panic disorder, generalized anxiety disorder, bipolar disorder, or psychosis). In the 1997 update of their seminal nationally representative estimates of complementary and alternative care use, Eisenberg et al. [8] found that $40.9 \%$ of individuals reporting severe depression and $42.7 \%$ of individuals with anxiety attacks used alternative therapy in the previous year. They estimated total expenditure on CAM for 1997 at $\$ 21.2$ billion, with estimated out-ofpocket expenditures totaling $\$ 9.1$ billion [9].

Grzywacz et al. [9] found that while CAM use was higher for the elderly with anxiety disorders, a majority did not use CAM to treat anxiety disorders. Su and $\mathrm{Li}$ [10] found CAM use was more likely among racial and ethnic minorities and when conventional treatment is more difficult. A number of meta-analyses have considered specific modality of CAM evaluated in a RCT structure. Chiesa and Serretti [11] found mindfulness-based stress reduction reduced ruminative thinking and trait anxiety, as well as increase empathy and selfcompassion. Manzoni et al. [12] found relaxation training showed a medium-large effect size in treatment in anxiety with higher efficacy for meditation and for longer treatments. Nestoriuc et al. [13] found biofeedback had significant effects in diminishing symptoms of anxiety and depression. Past cost-effectiveness analyses use randomized controlled trials or other quasi-experimental settings to examine the efficacy of specific complementary and alternative treatments or a narrow definition of CAM, finding generally favorable, but mixed results [14-16].

Bar-Sela et al. [17] use a pre-test and post-test design to assess the impact of CAM on a depression, anxiety, and fatigue in cancer patients undergoing oncology treatment, finding positive results on all dimensions. McPherson and McGraw [18] also used a pre-test and post-test design to assess the impact of CAM use on Generalized Anxiety Disorder for members of the military. Anxiety symptoms were improved for the study participants who completed the 6-week program.

\section{Objective}

The objective of this study is to assess the cost-effectiveness of complementary and alternative medicine (broadly defined) as addition to traditional treatments (drug therapy and psychotherapy) for anxiety disorders as measured by self-reported mental health status. Past costeffectiveness analyses have used randomized controlled trials (RCTs) to examine the efficacy of specific complementary and alternative treatments or a narrow definition of CAM.

This study uses the 1998 Medical Expenditure Panel Survey (MEPS), a panel survey of medical use, expenditure, and health status for the civilian non-institutionalized US population to investigate the outcomes for complementary and alternative medicine.

While past studies used this data to estimate use for different groups, none tried to estimate the cost-effectiveness. Davis and Darden examine the patterns of CAM use by children [19]. Ritchie et al. [20] examined the predictors of CAM use for a specific health concern. Neither of these studies documented differences in cost of effect of CAM use.

Unlike past studies of CAM that rely on an experimental (or quasiexperimental design), this study uses panel data combined with propensity score matching and inverse propensity score weighting in the incremental net benefit framework to estimate cost-effectiveness to uncover potential self-selection bias. The results demonstrate a minor impact on the cost-effectiveness estimates when adjusting for individual heterogeneity.

\section{Methods}

\section{Study design}

Data for the economic evaluation comes from the 1998 Medical Expenditure Panel Survey (MEPS), a nationally representative stratified random survey of the civilian noninstitutionalized population of the United States.

The MEPS is conducted by the Agency for Healthcare Research and Quality (AHRQ), part of the U.S. Department of Health and Human Services. The survey includes demographic characteristics, health conditions, health status, use of medical care services, charges and payments, access to care, satisfaction with care, health insurance coverage, income, and employment. Each household in a MEPS panel is interviewed for five rounds covering a two year period. Panel 2 covers calendar years 1997 and 1998 and panel 3 covers calendar years 1998 and 1999.

The Medical Expenditure Panel Survey (MEPS) uses a set of condition identifiers which aggregates clinically similar conditions by 5-digit DSM-IV TR Codes. The Anxiety Disorder condition identifier includes many different types of Anxiety Disorders. Perceived health status and perceived mental health status are both self-reported on a 5point Likert scale (1-Excellent, 2-Very Good, 3-Good, 4-Fair, and 5Poor). The MEPS also collects secondary, more specific measures of health status, including social limitations and cognitive limitations. This analysis considers an anxiety condition identifier based on clinically related DSM-IV-TR diagnosis of anxiety.

The 1998 MEPS includes a section of questions about complementary and alternative medicine (CAM). The MEPS definition of complementary and alternative medicine includes acupuncture, nutritional advice, massage therapy, herbal remedies, bio-feedback, imagery or relaxation techniques, homeopathic treatments, spiritual healing or prayer, hypnosis, or traditional medicine such as Chinese or American Indian medicine. Follow-up questions assess interaction with physician, use for specific problems, and spending and financing issues. 


\section{Cost Measurement}

The measure of cost for this analysis includes the direct total charges associated anxiety disorder. The four main sources of charges considered in this analysis are pharmacotherapy charges, office-based charges, outpatient-based charges, emergency room charges, and complementary and alternative medicine charges. Only the pharmacotherapy, office-based, emergency room-based, and outpatient-based charges linked to the specific anxiety condition identifier are considered under this analysis.

This analysis uses the measure of total charges in the Medical Expenditure Panel Survey (MEPS). As recommended, this is the broadest measure of cost, i.e., a societal measure of cost [21]. Direct costs are the primary measures of cost. Given limitations of the data available and evidence of similarities between CAM users and nonusers, the indirect costs associated with work incapacity were assumed negligible.

The MEPS identifies other sources of charges that are not considered as part of this analysis. The other sources of charges include home health-based charges, and dental visit-based charges, among others. Charges with mental health disorders from these sources would not represent typical treatment for the common mental health disorders under consideration, and thus, are excluded from this analysis.

\section{Outcome Measurement}

The primary measure of effectiveness of mental health treatment for this analysis is a global measure of self-reported mental health status. This global mental health measure would include changes in both physical and emotional health from the mental health disorders. The self-reported measure of mental health on a 5-point Likert scale (1Excellent, 2-Very Good, 3-Good, 4-Fair, and 5-Poor) considers any self-reported health status of "Good" or better as good mental health and "Fair" or "Poor" mental health are considered not good mental health. This dichotomous collapse of the 5-point self-reported mental health status is similar past research [22,23].

Secondary measures of mental health status for this analysis include self-reported limitations on instrumental activities of daily living, functional limitations, social limitations, and cognitive limitations. Limitations on the instrumental activities of daily living are measured by difficulty with using the telephone, paying bills, taking medication, preparing light meals, laundry, or shopping. Functional limitations are measured by difficulties walking, climbing stairs, grasping objects, reaching overhead, lifting, bending, stooping, or standing for long periods. Cogitative limitations are measured by confusion or memory loss, problems making decisions, to the point that it interferes with daily activities or requiring supervision for one's own safety. Social limitations are measured by limitations participating in social, recreational, or family activities.

There is a question of how meaningful self-reported global health measures are in terms of capturing clinically- or epidemiologicallymeaningful aspects of health.

In a review of studies related to the validity of self-rating of health, 23 of 27 studies found self-rating of health reliably predicted survival in populations even while accounting for known health risk factors [24]. Idler et al. [25] subsequently found self-rated health also predicts mortality and functions limitations using NHANES data.
Ried et al. [26] found that self-reported health status was moderately correlated with subjective well-being $=0.41, \mathrm{t}=11.3$, $\mathrm{p}<0.0001$ ), but the two were different constructs. Collapsing both measures to dichotomous variables also showed a statistically significant correlation $(0.63, \mathrm{p}<0.0001)$, but a difference in construct.

Global health measures include the Short Form-36 (SF-36) and the abridged Short Form-12 (SF-12). The SF-36 and SF-12 were surveys designed to capture significant variation in health domains. The eight domains of health in the SF-36 and SF-12 Index scores include physical functioning, role limitations because of physical health problems, bodily pain, social functioning, general mental health (psychological distress and psychological well-being), role limitations (from emotional problems), vitality (energy/fatigue), and general health perceptions.

The mental health scales of the SF-36 and SF-12 Index scores are based on the five-item Mental Health Inventory (MHI) that best predict summary score for the 38-item MHI, covering four major mental health dimensions (anxiety, depression, loss of behavioral or emotional control, and psychological well-being) [27]. The MHI has been successfully evaluated in clinical trials comparing quality of life outcomes and compared favorably with emotional reactions score from the Nottingham Health Profile and the summary SIP Psychosocial scale. The MHI discriminates psychiatric patients from those with other medical conditions [27].

The validity of the SF-36 Index score was investigated early in its development [28]. The authors found a good basis for establishing guidelines for the interpretation of score differences for each scale as a measure of physical and/or mental health effects. When observed differences are found in the physical functioning and mental health scales are found, physical or mental causes can be attributed with a high degree of confidence [28].

McCollum et al. [29] linked the SF-12 Index score to the selfreported health status measure used in this research for patients with diabetes and minor depression. Using the 2001 MEPS, they found that diabetes patients with minor depression had a lower mental health summary score (from SF-12), greater cognitive limitation, and lower mental health status as measured by a 5-point Likert scale controlling for relevant demographics and underlying health.

\section{Cost-effectiveness Assessment}

Cost-effectiveness analysis was calculated with the incremental costeffectiveness ratio (ICER) where the difference in cost $\left(\mu_{\Delta C}\right)$ between CAM users and CAM nonusers is normalized by the difference in effect between CAM users and CAM non users $\left(\mu_{\Delta \mathrm{E}}\right)$.

$$
\begin{aligned}
\text { ICER } & =\frac{\text { meancosts }_{C A M}-{ }_{\text {meancosts }}{ }_{\text {NoCAM }}}{\text { meaneffect } C A M-\text { meaneffect }}{ }_{\text {NoCAM }} \\
I C E R & =\frac{\mu_{\Delta C}}{\mu_{\Delta E}}
\end{aligned}
$$

A useful reformulation of the problem defines the incremental net benefit as the monetized difference in effect less the difference in the cost where $\lambda$ is the value of a unit of effect (either gained or lost) [30].

$$
\operatorname{INB}(\lambda)=\lambda * \mu_{\Delta E}-\mu_{\Delta C}
$$

A positive incremental net benefit is equivalent to a treatment being cost-effective and is evidence to support the adoption of the new 
Page 4 of 9

treatment in place of the control. The Cost-Effectiveness Acceptability Curve (CEAC) is a one way to convey the uncertainty of the costeffectiveness of treatment. Estimates of the CEAC can be obtained both through nonparametric bootstrapping repeated samples or using the p-values on the treatment coefficient from estimation of the incremental net benefit. The mirror image of the p-value (i.e., 1-(pvalue)) of the hypothesis test on a positive coefficient on the treatment dummy yields an estimate of the cost-effectiveness-acceptability curves that is asymptotically equivalent to the nonparametric bootstrapping approach [31]. Formally, the CEAC is defined as:

$$
\operatorname{CEAC}(\lambda)=\operatorname{Prob}\left(\operatorname{ICER}\langle\lambda| \mu_{\Delta E}>0\right)+\operatorname{Prob}\left(\operatorname{ICE} R\langle\lambda| \mu_{\Delta E}<0\right)
$$

The cost-effectiveness acceptability curve is interpreted as conditional probability of cost-effective CAM for a given value of effect.

\section{Investigating Self-Selection Bias}

Past cost-effectiveness analyses use randomized controlled trials to examine the efficacy of specific complementary and alternative treatments or a narrow definition of CAM. Unlike past costeffectiveness analyses that use randomized control trials, this analysis uses observational data to estimate cost-effectiveness. Individuals are not randomly assigned to either the control or treatment group, rather, they self-select into either control or treatment group. If the unobserved heterogeneity of the individual is correlated with the decision to select into either treatment or control group, then the estimates of differences in costs and effects (and, thus, incremental cost-effectiveness ratios) will be biased.

This research investigates the potential self-selection bias in two ways: inclusion of observable characteristics and inverse propensity score weighting. All are incorporated into the incremental net benefit framework. A simple extension of the basic incremental net benefit method includes a vector of observable characteristics $\left(\mathrm{X}_{\mathrm{i}}\right)$ :

$$
N B_{i}(\lambda)=\beta_{0}+\beta_{1} C A M_{i}+\Gamma X_{i}+\varepsilon_{i}
$$

Statistical differences in observable characteristics may be related to the unobservable heterogeneity that influences selection into either treatment or control. Propensity score method is recognized as a semiparametric approach to mitigate partially the effect of self-selection bias in non-experimental data [32]. All observations of CAM and CAM nonusers are used to estimate the predicted probability of treatment as a function of observable characteristics. The predicted probabilities of treatment for observations in the CAM group are matched to similar predicted probabilities of treatment for observation in the CAM nonuser group with a similar predicted probability using one-to-one nearest neighbor matching. Inverse propensity score weighting is similar to propensity score matching with the advantage that all observations of the sample are considered in the estimation of incremental net benefit. All observations in the CAM user and nonuser groups are used to estimate the predicted probability of treatment as a function of observable characteristics. The predicted probability of seeking treatment for an observation $\left(\mathrm{PS}_{\mathrm{i}}\right)$ is used to construct the weight for that observation according to the following:

$$
w_{i}=\frac{1}{P S_{i}}
$$

For observation i in the CAM group

$$
w_{i}=\frac{1}{1-P S_{i}} \text { For observation } \mathrm{i} \text { in the CAM nonuser group }
$$

Using inverse propensity score weighting in the estimation of incremental net benefit decreases the probability that CAM is costeffective for higher values of a unit of effect, except for the psychotherapy use sample. For low values of a unit of effect, using inverse propensity score weighting increases the probability that CAM is cost-effective.

\section{Statistical Analysis}

Baseline characteristics were analyzed using traditional Student ttests for comparing differences in continuous variables and a chisquared test for categorical variables. Incremental cost-effectiveness ratios and cost-effectiveness acceptability curves were estimated with multiple regressions including inverse propensity score weighting as described above. The significance level of 0.05 (two-sided) was used on baseline characteristics. SAS version 9.2 was used for estimation and bootstrapping cost-effectiveness analysis and the cost-effectiveness acceptability curves.

\section{Results}

\section{Baseline characteristics}

A total of 808 individuals with anxiety disorders were identified. Of those, $12.62 \%$ (102) were CAM users and $87.38 \%$ (706) were CAM nonusers.

As shown in Table 1, CAM users were more likely to be female and white, live in the west, be employed, and have at least some college.

Table 1 also presents the baseline characteristics of CAM users and nonusers. There are no statistically significant differences in baseline

\begin{tabular}{|c|c|c|c|c|c|}
\hline & CAM users & $(n=102)$ & CAM nonusers & $(n=706)$ & \\
\hline & MEAN & SD & MEAN & SD & p-Value \\
\hline \multicolumn{6}{|l|}{ Baseline Health Characteristics } \\
\hline Initial Overall Health (5pt Scale) & 2.70 & 1.21 & $2.87^{b}$ & 1.19 & 0.184 \\
\hline Initial Overall Mental Health (5pt Scale) & 2.64 & 1.12 & $2.66^{\mathrm{b}}$ & 1.14 & 0.823 \\
\hline Initial IADL Limitations & $0.0693^{a}$ & 0.2552 & $0.0939^{c}$ & 0.2919 & 0.850 \\
\hline
\end{tabular}
health characteristics between the two groups. CAM users are more likely to be female, white, live in the Northeast or West, and be employed. There were no statistically significant differences in income. 
Citation: Kutch M (2016) Cost-Effectiveness Analysis of Complementary and Alternative Medicine in Treating Anxiety Disorders. Altern Integr

\begin{tabular}{|c|c|c|c|c|c|}
\hline Initial Functional Limitations & 0.2255 & 0.4200 & $0.2340^{\mathrm{d}}$ & 0.4236 & 0.422 \\
\hline Initial Social Limitations & $0.1584^{a}$ & 0.3670 & $0.1280^{c}$ & 0.3344 & 0.399 \\
\hline Initial Cognitive Limitations & 0.1275 & 0.3351 & $0.1264^{\mathrm{e}}$ & 0.3326 & 0.977 \\
\hline \multicolumn{6}{|l|}{ Demographic Characteristics } \\
\hline Age & 43.67 & 13.78 & 47.53 & 15.86 & 0.196 \\
\hline Male & 0.1765 & 0.3831 & 0.3116 & 0.4635 & 0.005 \\
\hline White (Not Hispanic) & 0.8039 & 0.3990 & 0.7068 & 0.4556 & 0.041 \\
\hline Black (Not Hispanic) & 0.0588 & 0.2365 & 0.0949 & 0.2933 & 0.235 \\
\hline Hispanic & 0.1176 & 0.3238 & 0.1799 & 0.3844 & 0.120 \\
\hline Other Race (Not Hispanic) & 0.0196 & 0.1393 & 0.0184 & 0.1345 & 0.933 \\
\hline Northeast & 0.0686 & 0.2541 & 0.1856 & 0.3890 & 0.003 \\
\hline Midwest & 0.1765 & 0.3831 & 0.1926 & 0.3946 & 0.698 \\
\hline South & 0.3137 & 0.4663 & 0.3399 & 0.4740 & 0.600 \\
\hline West & 0.4412 & 0.4990 & 0.2819 & 0.4502 & 0.001 \\
\hline \multicolumn{6}{|c|}{ Socioeconomic Characteristics } \\
\hline Personal Income & 27776 & 26402 & 24269 & 25637 & 0.211 \\
\hline Employed & 0.6961 & 0.4622 & 0.5793 & 0.4940 & 0.025 \\
\hline College Graduate & 0.2353 & 0.4263 & 0.1856 & 0.3890 & 0.233 \\
\hline Other Degree & 0.0980 & 0.2988 & 0.0793 & 0.2704 & 0.519 \\
\hline Some College & 0.2157 & 0.4133 & 0.1827 & 0.3867 & 0.425 \\
\hline HS or Less & 0.4412 & 0.4990 & 0.5496 & 0.4979 & 0.040 \\
\hline
\end{tabular}

Table 1: Descriptive statistics for baseline characteristics.

\section{Cost Measurement}

Table 2 presents measures of cost and effectiveness between CAM users and nonusers. Office-based charges represent the largest source of traditional cost for both CAM users and nonusers $(\$ 141.29$ and $\$ 258.58$, respectively), followed by pharmaceutical costs ( $\$ 112.30$ and
\$167.11, respectively). CAM users have statistically significant lower levels of both office-based costs (p-value 0.022) and pharmaceutical costs (p-value 0.048 ) than CAM nonusers. The overall anxiety-related costs are not statistically difference ( $\$ 458.95$ versus $\$ 467.03$, p-value 0.920); this evidence implies that CAM users with anxiety disorders are substituting CAM treatment for both pharmacotherapy and psychotherapy treatment. (Table 3).

\begin{tabular}{|c|c|c|c|c|c|}
\hline & CAM users & $(n=102)$ & CAM nonusers & $(n=706)$ & \\
\hline & MEAN & SD & MEAN & SD & p-Value \\
\hline \multicolumn{6}{|l|}{ Outcomes } \\
\hline Overall Mental Health Status (5pt Scale) & 2.58 & 1.1383 & 2.70 & 1.1406 & 0.324 \\
\hline Probability [Good Mental Health] & 0.8039 & 0.3990 & 0.7720 & 0.4199 & 0.469 \\
\hline Probability [Steady or Improved Mental Health] & 0.7549 & 0.4323 & 0.6912 & 0.4623 & 0.190 \\
\hline
\end{tabular}


Citation: Kutch M (2016) Cost-Effectiveness Analysis of Complementary and Alternative Medicine in Treating Anxiety Disorders. Altern Integr

\begin{tabular}{|l|l|l|l|l|l|}
\hline Office-Based Costs & 141.29 & 323.14 & 258.58 & 1059.51 & 0.022 \\
\hline Outpatient-Based Costs & 0.64 & 6.44 & 21.18 & 320.31 & 0.089 \\
\hline ER-Based Costs & 2.21 & 20.45 & 20.16 & 169.10 & 0.007 \\
\hline Pharmaceutical Costs & 112.30 & 241.86 & 167.11 & 360.62 & 0.048 \\
\hline CAM Costs & 202.70 & 461.33 & 0.00 & 0.00 & $<0.001$ \\
\hline Total Costs & 458.95 & 657.56 & 467.03 & 1271.46 & 0.920 \\
\hline
\end{tabular}

Table 2: Measures of effectiveness and costs.

\begin{tabular}{|c|c|c|c|c|}
\hline & CAM users & CAM nonusers & Group Differences & p-Value \\
\hline Effect (in $\operatorname{Pr}[M H>=0]$ ) & 0.7549 & 0.6912 & 0.0637 & 0.190 \\
\hline Anxiety-Related Costs (mean) & 458.95 & 467.03 & -8.08 & 0.920 \\
\hline \multicolumn{5}{|l|}{ ICER } \\
\hline Estimated ICER (mean) & -1.27 & & & \\
\hline Quadrant & IV $(\Delta \mathrm{E}>0, \Delta \mathrm{C}<0)$ & & & \\
\hline Estimated ICER (median) & 24.06 & & & \\
\hline Quadrant & $\mathrm{I}(\Delta \mathrm{E}>0, \Delta \mathrm{C}>0)$ & & & \\
\hline WTP & $\operatorname{Pr}[\mathrm{CE} \mid \mathrm{WTP}]$ & $\operatorname{Pr}[C E \mid W T P, X]$ & $\operatorname{Pr}[\mathrm{CE}] \mathrm{w} / \mathrm{IPW}$ & \\
\hline 0 & 0.548 & 0.433 & 0.706 & \\
\hline 1000 & 0.786 & 0.706 & 0.885 & \\
\hline 2000 & 0.878 & 0.822 & 0.922 & \\
\hline 3000 & 0.909 & 0.862 & 0.930 & \\
\hline 4000 & 0.917 & 0.879 & 0.930 & \\
\hline 5000 & 0.920 & 0.887 & 0.931 & \\
\hline
\end{tabular}

Table 3: Incremental cost-effectiveness results.

\section{Outcome Measurement}

Three measures of effectiveness are considered. Average overall selfreported mental health on a 5-point Likert scale indicated CAM users ended with improved mental health (2.58 versus 2.70 ; $\mathrm{p}$-value 0.324$)$

The probability of ending with good mental health over the study period was higher for CAM users than for CAM nonusers $(0.8039$ versus 0.7720; $\mathrm{p}$-value 0.469).

The probability of steady or improved mental health over the study period was higher for CAM users than for CAM nonusers $(0.7549$ versus 0.6912; $\mathrm{p}$-value 0.190). A more ideal measure of effectiveness, Quality Adjusted Life Years (QALYs) would be used; unfortunately, this is not possible given the limitations of the data.

\section{Cost-Effectiveness Assessment}

This economic analysis shows that for those with anxiety, CAM users have slightly lower costs with higher effect than CAM nonusers, resulting in an ICER of $-\$ 1.27$ per $1 \%$ increase in the probability of steady or improved mental health. The difference in cost between groups is small, and not statistically significant.

The average difference in cost and effect between CAM users and nonusers is located in the quadrant IV of the incremental costincremental effectiveness plane (Figure 1).

These averages represent an increase in effectiveness $(0.0637$; $\mathrm{p}$ value 0.190$)$ and a decrease in cost (-\$8.08; p-value 0.920). Using median cost, the estimated ICER is $\$ 24.06$ per $1 \%$ increase in the probability of steady or improved mental health.

This difference in median cost would be located in quadrant I of the incremental cost-incremental effectiveness plane, with an increased effectiveness but also an increased cost. 


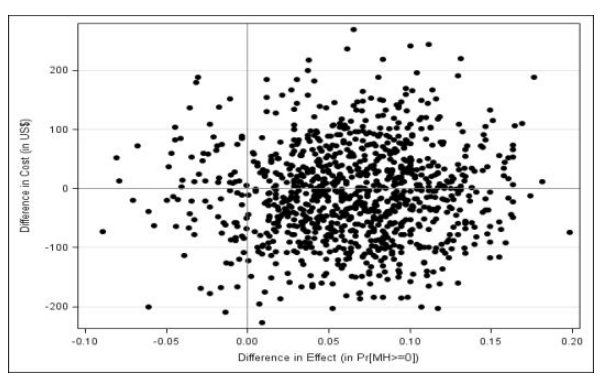

Figure 1: Bootstrap replicates of the difference in cost and effect in the incremental cost-incremental effectiveness space for CAM users versus nonusers. A significant portion are in quadrants I $(\Delta \mathrm{C}>0$, $\Delta \mathrm{E}>0)$ and $\mathrm{IV}(\Delta \mathrm{C}<0, \Delta \mathrm{E}>0)$.

To assess the uncertainty of the incremental cost-effectiveness ratio, Figure 1 shows 1000 bootstrapped replicates of the cost and effectiveness data. While individually, neither cost nor effectiveness is statistically significant at traditional levels of significance, it is clear that a substantial number of the replicates represent cost-effectiveness for a wide range of values of willingness-to-pay (WTP) for the effect. The cost-effectiveness acceptability curves based on the bootstrap replicates are a method to quantify the uncertainty of the incremental cost-effectiveness ratios. Figure 2 shows the cost-effectiveness acceptability curve for a range of values. The probability of a treatment being cost-effective for a given value of a unit of effect is estimated as the proportion of the 1,000 bootstraps that are cost-effective (i.e., below the value of a unit of effect line in the incremental costincremental effectiveness space). Values of a unit of effect (either the willingness-to-pay for greater effect or willingness-to-accept a lower level of effect) are measured on the horizontal axis and the probability that treatment is cost-effective is measured on the vertical axis.

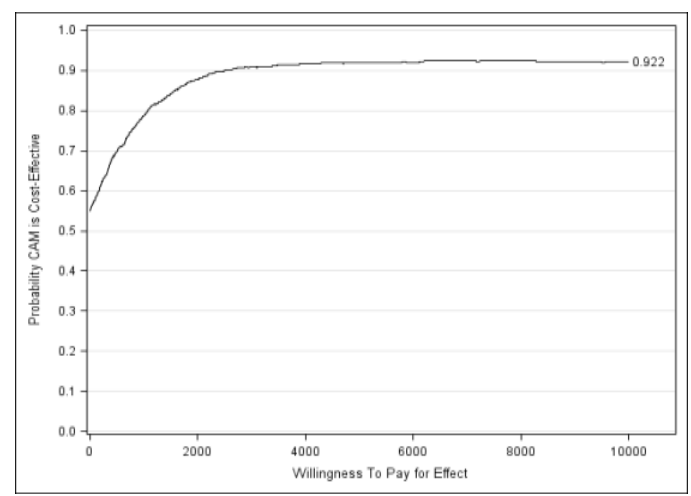

Figure 2: Cost-effectiveness acceptability curve showing probability CAM is cost-effective for a given willingness to pay for a unit of effect.

Including additional demographic controls into the estimation of incremental net benefit only slightly reduces the probability of costeffectiveness ( 0.920 reduced to 0.887 for $\mathrm{WTP}=5000$ for example). Additionally, using inverse propensity score weighting in the estimation of incremental net benefit slightly increases the probability that CAM is cost-effective for higher values of a unit of effect $(0.931$ versus 0.920 for $\mathrm{WTP}=5000$ for example) (Figure 3).

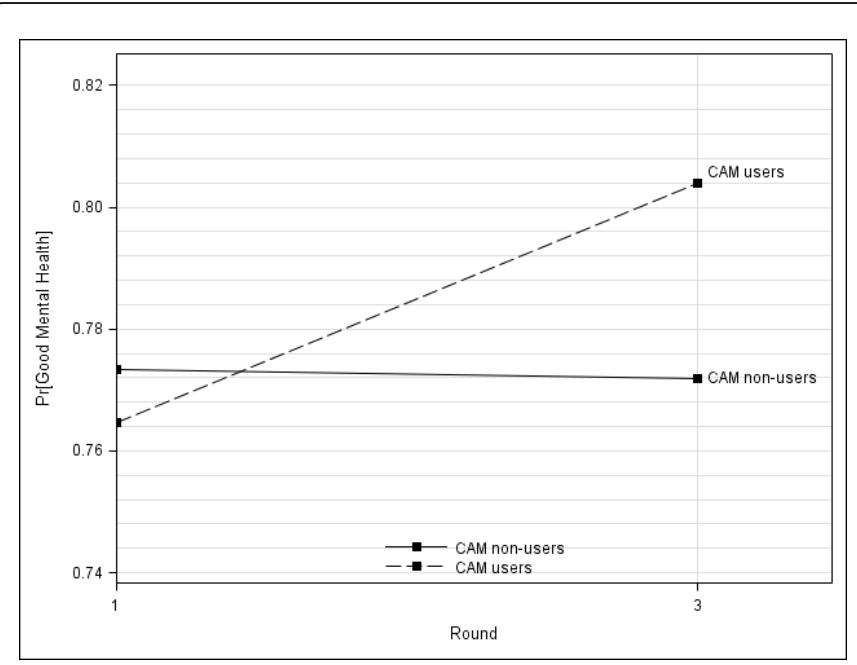

Figure 3: Probability of good mental health from initial measurement $(0.7647$ versus $0.7734 ; \mathrm{p}$-value 0.825$)$ to final measurement ( 0.8039 versus 0.7720 ; p-value 0.469$)$ for CAM users and nonusers.

\section{Discussion}

This research provides information on a very specific research question: Is complementary and alternative medicine (broadly defined) a cost-effective addition to traditional treatment for anxiety disorders the measures of the probability of good self-reported mental health status using observational data on patient treatment and characteristics? This research investigates potential self-selection bias of observational data in the estimates of the net benefit.

The most significant limitation of this current study is the age of the data. The current study extends the use the 1998 MEPS using current methods of cost-effectiveness analysis. However, if patterns of CAM use, patterns of anxiety treatment, and associated costs change significantly, then the current study should be interpreted as historical economic analysis describing the state of complementary and alternative medicine around the turn of the century. Su and $\mathrm{Li}$ [10] found an increase in CAM use in more current NHIS data, especially for chiropractics, massage, and acupuncture. While the extent to which the results apply to current CAM practices may be limited, these results still provide insight into the baseline of an analysis of CAM practices. If CAM costs increase at a faster rate than other forms of traditional therapy, the current results could represent a lower bound on the cost-effectiveness of CAM.

A second limitation of the current data concerns the definition of modalities included. The 1998 MEPS did not include prayer, spiritual beliefs, vitamins or supplements in the CAM definition. This explains the very significant difference in CAM use in this data compared to previous national estimates [5-8].

Another potential limitation of the current study is the use of selfreported mental health as a measure of effectiveness. While it is questionable if self-reported mental health status is reliable enough in measuring clinically significant changes in patients with anxiety, the direction of these results were statistically related in other MEPS years to improvement in other outcomes measures, specifically the EQ-5D 
Page 8 of 9

Index Score, the SF-12 Index Score, the probability of long-term work missed, and number of days missed.

Overall, these results suggest only a slight upward bias in the initial unconditional estimates of incremental net benefit for anxiety disorders. A positive correlation between seeking CAM treatment and the individual heterogeneity would produce this upward bias. If an individual knows that CAM will work better for them, they would be more likely to seek CAM treatment. If that individual heterogeneity is related to the observable characteristics, then controlling for them in the estimation of incremental net benefit will reduce this self-selection bias. The estimation of incremental net benefit using propensity matching is nearly identical to the unconditional estimation of incremental net benefit. These results suggest a slight bias in the initial unconditional estimates of incremental net benefit for anxiety disorder.

For anxiety disorders, there is some evidence to suggest that CAM is cost-effective for large values of a unit of effect. It should be noted that the values of a WTP associated with statistical significant of costeffectiveness may be too large, in practice, to justify; the opportunity cost of scarce healthcare dollars is not explicitly models and could, most likely, adversely impact the results. Efforts to investigate potential self-selection bias in the data show an upward bias in the initial, unconditional estimates of incremental net benefit.

This analysis also assumes that the use of charges instead of the cost of production does not bias the results in a systematic way. Implicitly, producer mark-up over the cost of production is assumed zero for both traditional and CAM treatment. Given the use of patent-protected pharmacotherapy treatments and the lack of perfectly competitive markets for each form of treatment, this assumption is questionable. However, it is not possible to assign an a priori expectation to this potential bias. Given the available data in the MEPS, there is not a feasible method for addressing this potential source of bias.

This analysis assumes that there is no difference in the level of direct nonmonetary resources and the indirect impact of treatment for CAM users and nonusers. The MEPS does include information regarding employment and compensation. If information on treatment acquisition time was available, it would be possible to construct a proxy for nonmonetary resource cost for this analysis. Most likely, acquisition time would vary by the of CAM treatment. Given the lack of detailed information on specific CAM treatment use, the lack of precise treatment acquisition time, and the limitations of employment compensation as a measure of time cost, this extension does not seem feasible for the current dataset.

Another possible route to investigate potential self-selection bias in observational data is with estimating net benefit with instrumental variables. This would require an instrument that is correlated with the decision to seek CAM treatment that is uncorrelated with the outcome of CAM treatment. State-level differences in reimbursement rates could serve as another potential instrument in future research. However, state identifiers are not available in the publically available MEPS used in this analysis.

\section{Conclusion}

In conclusion, this observational study indicates for patients with anxiety disorders CAM users have improved outcomes and slightly lower costs with a high probability of cost-effectiveness. While these findings working with the best available tools in the 1998 MEPS are interesting, certainly additional research is warranted, especially given the data limitations and shortcomings. Future research would benefit from collecting cost information alongside RCTs of different CAM modalities with better established measurements of anxiety disorders.

\section{Acknowledgement}

This work was begun as part of a dissertation requirement. The author thanks Alvin Headen, Stephen Margolis, Tamah Morant, and Melinda Morrill for helpful feedback throughout the stages of this project. All errors are the author's own. The author would also like to thank the anonymous reviewers for their helpful feedback.

\section{References}

1. Grimaldi-Bensouda L, Begaud B, Lert F, Rouillon F, Massol J, et al. (2011) Benchmarking the burden of 100 diseases: results of a nationwide representative survey within general practices. BMJ Open 1: e000215.

2. Kessler RC, Chiu WT, Demler O, Merikangas KR, Walters EE (2005) Prevalence, severity, and comorbidity of 12-month DSM-IV disorders in the National Comorbidity Survey Replication. Arch Gen Psychiatry 62: 617-627.

3. Mark TL, Levit KR, Yee T, Chow CM (2014) Spending on mental and substance use disorders projected to grow more slowly than all health spending through 2020. Health Aff (Millwood) 33: 1407-1415.

4. National Institute of Mental Health (2016) Anxiety disorders.

5. Barnes PM, Bloom B, Nahin RL (2008) Complementary and alternative medicine use among adults and children: United States, 2007. Natl Health Stat Report 1-23.

6. Kessler RC, Soukup J, Davis RB, Foster DF, Wilkey SA, et al. (2001) The use of complementary and alternative therapies to treat anxiety and depression in the United States. Am J Psychiatry 158: 289-294.

7. Unützer J, Klap R, Sturm R, Young AS, Marmon T, et al. (2000) Mental disorders and the use of alternative medicine: results from a national survey. Am J Psychiatry 157: 1851-1857.

8. Eisenberg DM, Davis RB, Ettner SL, Appel S, Wilkey S, et al. (1998) Trends in alternative medicine use in the United States, 1990-1997: results of a follow-up national survey. JAMA 280: 1569-1575.

9. Grzywacz JG, Suerken CK, Quandt SA, Bell RA, Lang W, et al (2006) Older adults' use of complementary and alternative medicine for mental health: findings from the 2002 National Health Interview Survey. J Altern Complement Med 12: 467-473.

10. Su D, Li L (2011) Trends in the use of complementary and alternative medicine in the United States: 2002-2007. J Health Care Poor Underserved 22: 296-310.

11. Chiesa A, Serretti A (2009) Mindfulness-based stress reduction for stress management in healthy people: a review and meta-analysis. J Altern Complement Med 15: 593-600.

12. Manzoni GM, Pagnini F, Castelnuovo G, Molinari E (2008) Relaxation training for anxiety: a ten-years systematic review with meta-analysis. BMC Psychiatry 8: 41.

13. Nestoriuc Y, Martin A, Rief W, Andrasik F (2008) Biofeedback treatment for headache disorders: a comprehensive efficacy review. Appl Psychophysiol Biofeedback 33: 125-140.

14. Solomon D, Adams J, Graves N (2013) Economic evaluation of St. John's wort (Hypericum perforatum) for the treatment of mild to moderate depression. J Affect Disord 148: 228-234.

15. Canter PH, Coon JT, Ernst E (2005) Cost effectiveness of complementary treatments in the United Kingdom: systematic review. BMJ 331: 880-881.

16. Herman PM, Poindexter BL, Witt CM, Eisenberg DM (2012) Are complementary therapies and integrative care cost-effective? A systematic review of economic evaluations. BMJ Open 2: e001046.

17. Bar-Sela G, Danos S, Visel B, Mashiach T, Mitnik I (2015) The effect of complementary and alternative medicine on quality of life, depression, 
Citation: Kutch M (2016) Cost-Effectiveness Analysis of Complementary and Alternative Medicine in Treating Anxiety Disorders. Altern Integr

Page 9 of 9

anxiety, and fatigue levels among cancer patients during active oncology treatment: phase II study. Support Care Cancer 23: 1979-1985.

18. McPherson F, McGraw L (2013) Treating generalized anxiety disorder using complementary and alternative medicine. Altern Ther Health Med 19: $45-50$.

19. Davis MP, Darden PM (2003) Use of complementary and alternative medicine by children in the United States. Arch Pediatr Adolesc Med 157: 393-396.

20. Ritchie CS, Gohmann SF, McKinney WP (2005) Does use of CAM for specific health problems increase with reduced access to care? J Med Syst 29: 143-153.

21. Gold M, Siegel J, Russell L, Weinstein M (1996) Cost-effectiveness in health and medicine. New York: Oxford University Press, USA.

22. Spinks J, Hollingsworth B (2012) Policy implications of complementary and alternative medicine use in Australia: data from the National Health Survey. J Altern Complement Med 18: 371-378.

23. Tait EM, Laditka JN, Laditka SB, Nies MA, Racine EF, et al (2013) Reasons why older americans use complementary and alternative medicine: Costly or ineffective conventional medicine and recommendations from health care providers, family, and friends. Educ Gerontol 39: 684-700.

24. Idler EL, Benyamini Y (1997) Self-rated health and mortality: a review of twenty-seven community studies. J Health Soc Behav 38: 21-37.

25. Idler EL, Russell LB, Davis D (2000) Survival, functional limitations, and self-rated health in the NHANES I Epidemiologic Follow-up Study, 1992.
First National Health and Nutrition Examination Survey. Am J Epidemiol 152: 874-883.

26. Ried LD, Tueth MJ, Handberg E, Nyanteh H (2006) Validating a selfreport measure of global subjective well-being to predict adverse clinical outcomes. Qual Life Res 15: 675-686.

27. Ware J, Sherbourne C (1992) The MOS 36-item short-form health survey (SF-36) I: Conceptual framework and item selection. Med Care 30: 473-483.

28. McHorney CA, Ware JE Jr, Raczek AE (1993) The MOS 36-Item ShortForm Health Survey (SF-36): II. Psychometric and clinical tests of validity in measuring physical and mental health constructs. Med Care 31: 247-263.

29. McCollum M, Hansen LB, Ghushchyan V, Sullivan PW (2007) Inconsistent health perceptions for US women and men with diabetes. J Womens Health (Larchmt) 16: 1421-1428.

30. Stinnett AA, Mullahy J (1998) Net health benefits: a new framework for the analysis of uncertainty in cost-effectiveness analysis. Med Decis Making 18: S68-S80.

31. Fenwick E, O'Brien B, Briggs A (2004) Cost-effectiveness acceptability curves - facts, fallacies, and frequently asked questions. Health Econ 13: 405-415.

32. Dehejia R, Wahba S (2002) Propensity score-matching methods for nonexperimental causal studies. Rev Econ Stat 84: 151-161. 